\title{
DESIGN OF TUNED MASS DAMPERS FOR LARGE STRUCTURES USING MODAL ANALYSIS
}

\author{
JAN ŠTĚPÁNEK*， JiŘí MÁCA \\ Czech Technical University in Prague, Faculty of Civil Engineering, Department of Mechanics, Thákurova 7, \\ 16629 Prague 6, Czech Republic \\ * corresponding author: jan.stepanek@fsv.cvut.cz
}

\begin{abstract}
Tuned mass damper is a device, which can be highly useful when dealing with excessive vibration and is widely used in many engineering fields. However, its proper design and optimization is a complicated task. This study uses mode superposition method to speed up the evaluation of dynamic response. The speed of response calculation allows for a quick calculation of frequency response function and numerical optimization of tuned mass dampers. This optimization method is demonstrated on a numerical example of a cable stayed footbridge. The example compares a simplified and widely used design method of tuned mass damper with numerical optimization.
\end{abstract}

KEYWORDS: Dynamic response, modal coordinates, tuned mass damper, optimization.

\section{INTRODUCTION}

The tuned mass damper (TMD) is a device composed of a spring, mass and a viscous damper, which is widely used in order to reduce mechanical vibration. However, its high efficiency is conditioned by appropriate design. Unfortunately, the closed form solution for its optimal parameters has been found only for special, simplified cases.

Numerous studies dealing with optimal design of TMD have been published since the beginning of the 20th cetury, but most of them only consider a single dergee of freedom (SDOF) main structure. The first design method for stifness of TMD spring was proposed by Den Hartog [1. It comes from observation of two invariant points on frequency response function (FRF), which are independent of TMD damping. The optimal damping was derived by Brock [2] for both motion and force excitation. These settings of parameters are close to the optimal ones for undamped main structure. Thanks to simple application, it is still a recomended design method in several modern guidelines [3 5]. The closed form solution of optimal tuning and damping for undamped main SDOF system was found by O. Nishihara and T. Asami [6]. Abubakar and Farid presented a numericaly derived formula for design of TMD on clasically damped SDOF structure [7].

Simplification of main structure by SDOF model is sometimes insufficient and may lead to wrong tuning or unnecessary big mass of TMD. Especially, large structures such as bridges or high buildings, which may be sensitive to dynamic load, cannot be modeled as SDOF structures. Rana and Soong showed the importance of numerical tuning in case of multiple degrees of freedom (MDOF) structures and shows the example of multiple TMDs, each tuned for particular mode [8]. Ozer and Royston extended Hartog's idea to MDOF systems [9, but the proposed method re- quires inversion of the dynamic stiffness matrix and numerical solution of system of equations, which may be impractical.

This paper presents numerical optimization of single and multiple TMDs, on a MDOF damped stucture. The goal is to minimize the maximal amplitude (also called $H_{\infty}$ optimization) of the structure caused by simple harmonic load. In the case of $H_{\infty}$ numerical optimization, the problem is that large amount of solutions needs to be evaluated for various TMD parameter values and forcing frequencies. To reduce the camputational complexity of the problem, method proposed in [10] was applied. This method operates with transformation to modal coordinates and allows us to find steady-state response of structures with TMD without solving a system of equations. This method is also suitable for design of TMDs for existing structures where excessive vibrations were measured and modal characteristics are known.

\section{Response CALCULATiON}

The main structure is represented by classically damped MDOF system with $n$ degrees of freedom and it is loaded by general dynamic load $F(t)$. The system is described by equation of motion

$$
K x+C \dot{x}+M \ddot{x}=F(t),
$$

where $K, C$ and $M$ represent stiffness matrix, damping matrix, and mass matrix, respectively, $F(t)$ is the loading vector and $x$ is vector of displacement. The vectors of velocity and acceleration are represented by symbols $\dot{x}$ and $\ddot{x}$.

\subsection{SINGLE TMD}

TMD is attached to the $m$-th degree of freedom. The total system can be represented by system of $n+1$ equations, but the equation which corresponds to the 
mass of TMD can be separated [9], as can be seen in Eqs. (2) and (3).

$$
\begin{aligned}
& {\left[K+K^{a}\right] x+\left[C+C^{a}\right] \dot{x}+M \ddot{x}} \\
& \quad-K_{m}^{a} x_{a}-C_{m}^{a} \dot{x}_{a}=F(t), \\
& k_{a} x_{a}+c_{a} \dot{x}_{a}+m_{a} \ddot{x}_{a}-\left[C_{m}^{a}\right]^{T} \dot{x}-\left[K_{m}^{a}\right]^{T} x=0,
\end{aligned}
$$

where:

$K^{a} \quad$ defined by Eq. (4),

$C^{a}$ defined by Eq. (5),

$K_{m}^{a} \quad m$-th column of matrix $K^{a}$,

$C_{m}^{a} \quad m$-th column of matrix $C^{a}$,

$k_{a} \quad$ stiffness of TMD,

$c_{a} \quad$ damping of TMD,

$m_{a}$ mass of TMD,

$x_{a} \quad$ displacement of TMD,

$\dot{x}_{a} \quad$ velocity of TMD,

$\ddot{x}_{a} \quad$ acceleration of TMD.

Matrices $K^{a}$ and $C^{a}$ contain only a single non-zero element on the $m \times m$ position:

$$
\begin{gathered}
K^{a}=\left[\begin{array}{cccc}
0 & 0 & \cdots & 0 \\
0 & \ddots & \vdots & \vdots \\
\vdots & \cdots & k_{a(m \times m)} & \vdots \\
0 & \cdots & \cdots & 0
\end{array}\right], \\
C^{a}=\left[\begin{array}{cccc}
0 & 0 & \cdots & 0 \\
0 & \ddots & \vdots & \vdots \\
\vdots & \cdots & c_{a(m \times m)} & \vdots \\
0 & \cdots & \cdots & 0
\end{array}\right] .
\end{gathered}
$$

Assuming the loading force is harmonic with amplitude $F_{a}$ and circular frequency $\omega$, we only need to find a solution in the form of steady-state response. The equations (2) and (3) can be transformed to modal coordinates according to Eq. (7). Substituting $x_{a}$ expressed from Eq. (3) to Eq. (2), the following result is obtained:

$$
\begin{aligned}
& {\left[\Omega+\mathrm{i} \omega C^{\prime}-\omega^{2} \mathrm{I}+\right.} \\
& \left.+\frac{\left(\mathrm{i} \omega c_{a}+k_{a}\right)\left(-\omega^{2} m_{a}\right)}{-\omega^{2} m_{a}+\mathrm{i} \omega c_{a}+k_{a}} \Phi_{m, *}^{T} \Phi_{m, *}\right] q=\Phi^{T} F_{a}, \\
& x=\Phi q,
\end{aligned}
$$

where $\Omega, \Phi$, and $C^{\prime}$ are spectral matrix, modal matrix, and modal damping matrix, respectively. $\Phi_{m, *}$ denotes the $m$-th row of modal matrix and $q$ is the vector of modal coordinates. The modal matrix must be mass orthonormal with mode shapes $\phi$ arranged as its columns. The spectral matrix contains squares of natural circular frequencies $\omega_{j}$ on its diagonal. For a classically damped structure, $C^{\prime}$ is also diagonal containing members $2 \xi_{j} \omega_{j}$ where $\xi_{j}$ is the damping ratio of $j$-th natural frequency.

Eq. (6) is a system of $n$ linear equations. Thanks to its structure, Sherman-Morrison formula can be used to find the inversion of the matrix on the left-hand side. The solution of separate modal coordinate $q_{j}$ with used substitutions is given by following equations:

$$
\begin{gathered}
{\left[\frac{\omega^{2} m_{a}\left(\mathrm{i} \omega c_{a}+k_{a}\right) \Phi_{m, j} S_{2}^{T}}{\left(-\omega^{2} m_{a}+\mathrm{i} \omega c_{a}+k_{a}\right)-\omega^{2} m_{a}\left(\mathrm{i} \omega c_{a}+k_{a}\right) s_{1}}+\right.} \\
\left.+\phi_{j}^{T}\right] \frac{F_{A}}{\omega_{j}^{2}-\omega^{2}+2 \mathrm{i} \xi_{j} \omega \omega_{j}}=q_{j} \\
s_{1}=\sum_{j=1}^{n} \frac{\Phi_{m, j}^{2}}{\omega_{j}^{2}-\omega^{2}+2 \mathrm{i} \xi_{j} \omega \omega_{j}} \\
S_{2}^{T}=\sum_{j=1}^{n} \frac{\Phi_{m, j} \phi_{j}^{T}}{\omega_{j}^{2}-\omega^{2}+2 \mathrm{i} \xi_{j} \omega \omega_{j}}
\end{gathered}
$$

As can be seen in Eq. 8 10, all mode shapes must be known to find an exact contribution of one particular mode shape to the response. However, mode shapes with frequencies, which are far from forcing frequency have negligible influence on the response. Therefore using only several selected mode shapes in Eqs. $7 \sqrt{10}$ can lead to very precise results. The reduction of number of mode shapes is necessary for fast response evaluation.

\subsection{MULTIPLE TMDS}

In the case of multiple TMDs, the evaluation of separate modal coordinates using Eq. (8) is no longer possible, but Sherman-Morrison formula

$$
A_{i+1}^{-1}=\left[A_{i}+u v^{T}\right]^{-1}=A_{i}^{-1}-\frac{A_{i}^{-1} u v^{T} A_{i}^{-1}}{1+v^{T} A_{i}^{-1} u}
$$

can be repeatedly applied to get a vector of modal coordinates $q$. Each addition of TMD requires one evaluation of Eq. (11) with substitutions given by Eqs. 12, 14.

$$
\begin{gathered}
A_{0}=\Omega+\mathrm{i} \omega C^{\prime}-\omega^{2} \mathrm{I}, \\
u=\frac{\left(\mathrm{i} \omega c_{a}+k_{a}\right)\left(-\omega^{2} m_{a}\right)}{-\omega^{2} m_{a}+\mathrm{i} \omega c_{a}+k_{a}} \Phi_{m, *}^{T} . \\
v^{T}=\Phi_{m, *}
\end{gathered}
$$

For $j$ TMDs, the vector of modal coordinates is

$$
q=A_{j}^{-1} \Phi^{T} F_{a} .
$$

This method may be inappropriate for large number of TMDs because of its high numerical complexity caused by multiplication of full matrices. However, its usage still avoids the solution of the system of 
equations. In section 4.5 this method is successfully used for the evaluation of structural response with three TMDs.

As can be seen in Eqs. (11,14), the system receptance matrix $A_{j}^{-1}$ is found without necessity of matrix inversion. The only matrix which must be inverted is $A_{0}$, but it is a simple procedure, because $A_{0}$ is a diagonal matrix. The receptance matrix can be used for fast evaluation of response to various load vectors.

\section{SimplifiEd TMD DESIGN}

Well known simplified design of TMD for minimization of maximal amplitude, first introduced in [1, 2] by Hartog and Brock, is used in this study as reference method and is compared to numerical optimization of TMDs. This method was chosen for its easy application and the fact it is widely used and recommended.

Using following notation:

$\begin{array}{ll}M_{e f f} & \begin{array}{l}\text { effective mass of the main struc- } \\ \text { ture, } \\ \end{array} \\ \Omega_{j} \text {-th natural frequency of the } \\ & \text { main structure, } \\ \omega_{a}=\sqrt{k_{a} / m_{a}} & \text { natural frequency of TMD, } \\ \mu=m_{a} / M_{e f f} & \text { mass ratio, } \\ \beta=\omega_{a} / \Omega_{j} & \text { frequency ratio, } \\ \xi_{a}=c_{a} / c_{a, c r} & \text { damping ratio of TMD, } \\ c_{a, c r}=2 \sqrt{k_{a} m_{a}} & \text { critical damping of TMD. }\end{array}$

the optimal values of parameters $\beta$ and $\xi_{a}$ for force excitation are

$$
\begin{gathered}
\beta_{o p t}=\frac{1}{1+\mu}, \\
\xi_{a, o p t}=\sqrt{\frac{3 \mu}{8(1+\mu)}} .
\end{gathered}
$$

This method was firstly proposed for SDOF main structure, but it can be used for design of TMD which is supposed to reduce vibration of $j$-th mode shape. The effective mass of the main structure $M_{\text {eff }}$ can be found according to the following equation:

$$
M_{e f f}=\frac{1}{\Phi_{m, j}^{2}}=\phi_{j}^{\prime T} M \phi_{j}^{\prime},
$$

where $\phi_{j}^{\prime}$ denotes the $j$-th mode shape normalized with respect to the $m$-th ordinate where TMD is attached hence the $m$-th ordinate is equal to one.

\section{Numerical exAmple}

This chapter analysis a cable stayed footbridge. The structure is suspected of being sensitive to dynamic pedestrian load. A Numerical model of the structure was created in MATLAB. Design of TMDs was performed both using simplified method indicated in section 3 and numerical optimization.

\begin{tabular}{ccc}
\hline mode & frequency $[\mathrm{Hz}]$ & damping ratio $[-]$ \\
\hline 1 & 0.733 & 0.0190 \\
2 & 0.923 & 0.0148 \\
3 & 1.300 & 0.0106 \\
4 & 1.595 & 0.0087 \\
5 & 1.818 & 0.0078 \\
6 & 1.924 & 0.0075 \\
7 & 2.002 & 0.0071 \\
8 & 2.218 & 0.0063 \\
\hline
\end{tabular}

TABLE 1. Natural frequencies and damping ratios.

\subsection{Model of the Structure}

The structure is $243 \mathrm{~m}$ long, symmetric, cablestayed bridge with prestressed concrete bridge deck. A scheme of the structure and numbering of important nodes can be seen in Figure 1 Two-dimensional finite element (FEM) model was created in order to examine vertical vibrations. The model contains nodes which define geometry of the structure and also uses nodes added by FEM mesh. The final model contains 810 degrees of freedom.

Eight lowest natural frequencies, mode shapes and associated damping ratios were measured on the real structure. Only vertical bending mode shapes are taken into account in this paper. The numerical model was identified to be in accordance with measurement. Natural frequencies and damping ratios are summarized in Table 1

\subsection{LOAD}

As can be seen in Table 1, several frequencies are close to $2 \mathrm{~Hz}$, which is typical frequency of human walk and thus resonance effect may occure. According to guidebook [5], a stationary load model which represents a group of 8-15 walkers can be used to evaluate the response and to decide whether the vibration is excessive. The group of walkers is simplified to one harmonic force in resonance with mode, which has the frequency closest to $2 \mathrm{~Hz}$. The force should be applied to the most adverse position, which may be understood as a place with the highest vertical ordinate of the forced mode shape.

In the case given, it was decided to find maximal displacement over the bridge span for three positions, which corresponds to the maximal ordinate of mode shapes. They are node 19 for the 5 th mode shape, node 5 for the 6 th, and node 27 for the 7 th one. The results can be seen in Figure 1 The highest peak in range between $1.5-2.5 \mathrm{~Hz}$ is caused by force situated in node 5 in resonance with 6 th natural frequency. Therefore the harmonic force with amplitude $f_{v}$ is placed in node 5 .

$$
f_{v}=k_{v} \times 180=3 \times 180=540 \mathrm{~N} .
$$

However, the amplitude $f_{v}$ is important only for taking the absolute value of the response, but it has no impact on the design of TMD. 


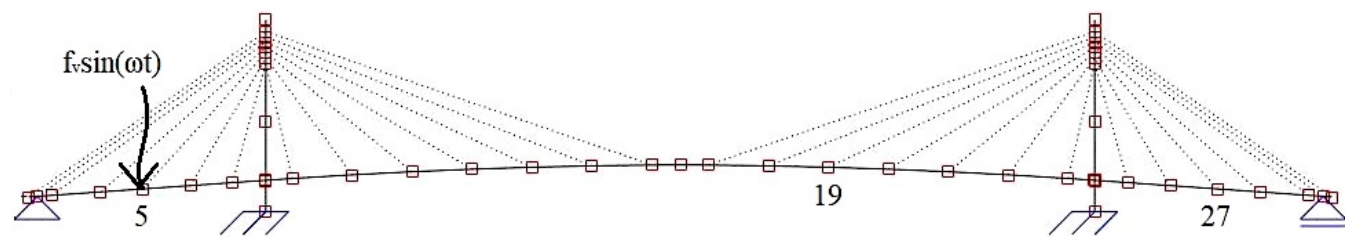

Figure 1. Static scheme of the bridge.

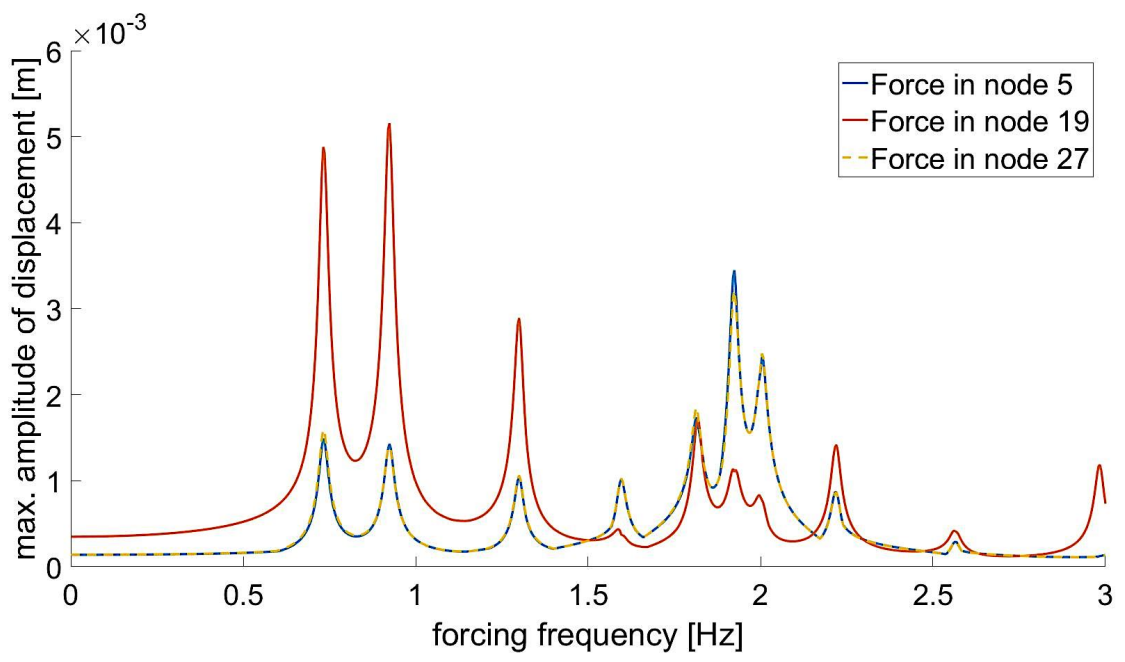

Figure 2. FRFs of force in nodes 5,19, and 27.

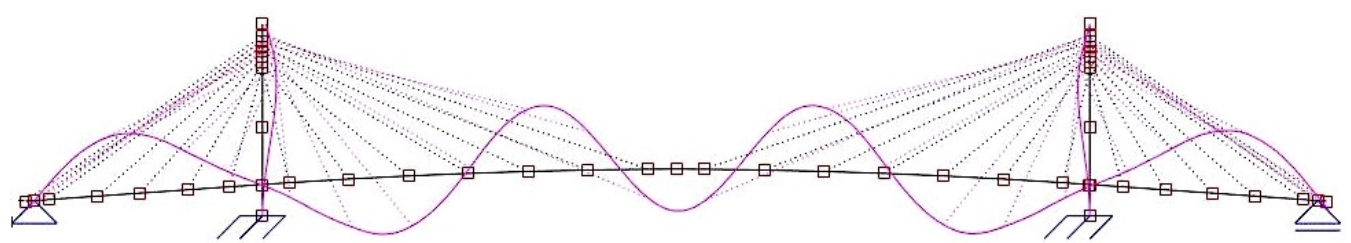

Figure 3. Mode shape No. 5.

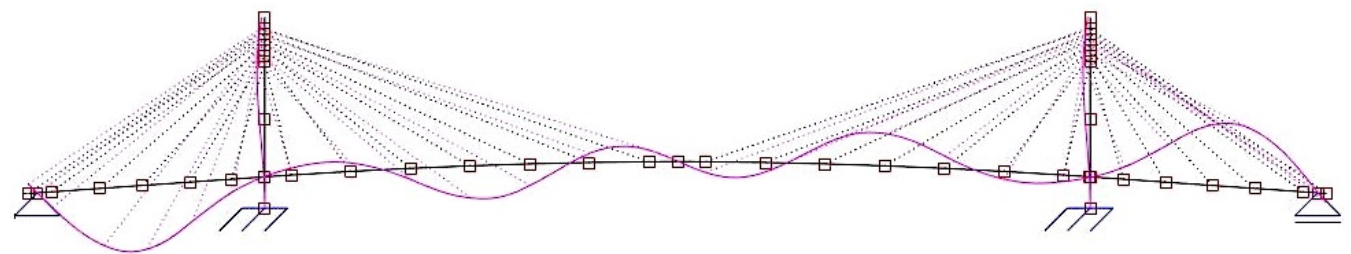

Figure 4. Mode shape No. 6.

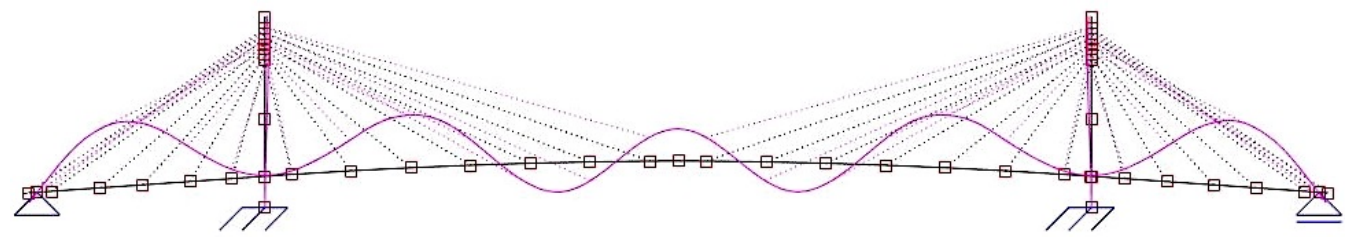

Figure 5. Mode shape No. 7.

Node 5 is appropriate place for simple harmonic load, because mode shapes 5,6 and 8 also have high ordinate in this node, therefore multiple modes are forced. For simplified TMD design from section 3 the load does not play a role, but it is important for numerical optimization and calculation of FRF.

\subsection{OBJECTIVE FUNCTION}

An objective function must be defined for the optimization of TMD. In this paper, it was decided to use $H_{\infty}$ optimization - maximal value of displacement amplitude along the bridge deck between 1.7 and $2.2 \mathrm{~Hz}$, where the load causes highest response. That means 
maximal values of vector of displacement $x$ create FRF, which creates a continuous FRF with discontinuous derivative. Highest peak of FRF is then the value of the objective function which is minimized. The step of forcing frequency for evaluating the FRF was set to $0.001 \mathrm{~Hz}$ to catch the sharp peaks but with respect to the speed of calculation.

The transformation to modal coordinates allows us to use reduced number of mode shapes to speed up the evaluation of response. Observation of FRF showed that using more than first 12 mode shapes and natural frequencies does not affect the values of peak response in chosen frequency range. Therefore it was decided to use only first 12 mode shapes. The reduction of number of mode shapes reduced the size of the problem dramatically (from 810 equations to 12 equations) without noticeable decrease of accuracy.

\subsection{SINGLE TMD}

\subsubsection{SIMPLIFIED DESIGN}

The simplified design method presented in section 3 was performed in order to reduce vibration of the sixth mode shape, because the pacing force causes the highest peak on FRF in resonance with the sixth natural frequency, as can be seen in Figure 2

TMD with one vertical degree of freedom and mass $m_{a}=600 \mathrm{~kg}$ is attached to node 5 , and its parameters were designed according to section 3 which resulted into values $k_{a}=86300 \mathrm{Nm}^{-1}$, and $c_{a}=772 \mathrm{Nsm}^{-1}$. The mass of $600 \mathrm{~kg}$ is approximately only $0.15 \%$ of the total mass of the structure, but it can reduce the vibration significantly.

\subsubsection{NUMERICAL OPTIMIZATION}

To optimize TMD numerically, function fmincon implemented in MATLAB was used. The function uses interior-point algorithm and is designed to solve constrained multivariable optimization problems. The only implemented constraints were $k_{a}>0$ and $c_{a}>0$. The function was used to find optimal values of $k_{a}$ and $c_{a}$ which minimize the objective function. Generally, in the case of single TMD, the objective function can have at most one local minimum for each natural frequency. In our case, the function has only one local minimum for the sixth frequency, because the peak of FRF reaches the highest level in resonance with the sixth natural frequency.

The initial values of $k_{a}$ and $c_{a}$ define the local minimum to which interior-point algorithm converges, therefore it is advantageous to begin with TMD tuned close to the natural frequency which response is minimized. For parameters of TMD, which are "far from optimal", a gradient of objective function may be very low, therefore convergence problems may occur, or it can take a large number of iterations to find the local minimum. Parameters designed according to section 3 are quality starting point for quick convergence.

The parameters, which optimize the objective function $k_{a}=84100 \mathrm{Nm}^{-1}$ and $c_{a}=1259 \mathrm{Nsm}^{-1}$ were

\begin{tabular}{ccccc}
\hline $\begin{array}{c}\text { mode } \\
\text { shape }\end{array}$ & node & $\begin{array}{c}k_{a} \\
{\left[\mathbf{N m}^{-1}\right]}\end{array}$ & $\begin{array}{c}c_{a} \\
{\left[\mathbf{N s m}^{-1}\right]}\end{array}$ & $\begin{array}{c}m_{a} \\
{[\mathbf{k g}]}\end{array}$ \\
\hline 5 & 19 & 26000 & 98 & 200 \\
6 & 5 & 29100 & 150 & 200 \\
7 & 27 & 31500 & 129 & 200 \\
\hline
\end{tabular}

TABLE 2. TMDs designed to reduce vibration of the 5 th, 6th, and 7th mode shape.

found numerically. Corresponding FRFs for both methods of design can be seen in Figure 6 .

\subsection{Multiple TMDs}

As we can see in Figure 2, there are three dominant natural frequencies between range 1.7-2.2 Hz. Therefore three TMDs are designed in this section to show that a higher number of TMDs can reduce the objective function more effectively than one. It was decided to keep the sum of TMDs mass constantly at $600 \mathrm{~kg}$. The constant sum of weight allows for a relevant comparison of one and multiple TMDs.

\subsubsection{SIMPLIFIED DESIGN}

In order to reduce FRF, three tuned mass dampers were designed according to section 3 to reduce vibration in resonance with the 5 th, 6 th, and 7 th natural frequencies. All of them were positioned to the node where the highest ordinate of damped mode is located. Their properties are summarized in Tab. 2 The mass was equally distributed among TMDs because its optimal distribution remains unknown.

\subsubsection{NUMERICAL OPTIMIZATION}

The numerical optimization was performed with two basic settings. The first one uses position of TMDs used by previous section (nodes 19, 5, and 27) and was expected to provide the best results. The second one places all TMDs in node 5. This calculation was proceeded in order to show that dividing the mass among more TMDs can perform better than one TMD, if more than one mode participate on the response. Theoretically, one TMD is only a subset of multiple TMDs with the same sum of mass. Therefore, dividing the mass provided additional room for a performance improvement.

The objective function was minimized finding $c_{a, i}$, $k_{a, i}$, and $m_{a, i}$ for $\mathrm{i}=1 \ldots 3$. The following constraints were implemented:

- $c_{a, i} \geq 0$

- $k_{a, i} \geq 0$,

- $m_{a, i} \geq 0$,

- $\sum_{i=1}^{3} m_{a, i}=600 \mathrm{~kg}$.

From the numerical point of view, the problem is much more complicated than the one with single TMD because 9 variables were optimized. Moreover, the function contains multiple local minima. Therefore, 


\begin{tabular}{|c|c|c|c|c|c|c|}
\hline settings No. & design method & node & $\begin{array}{c}k_{a} \\
{\left[\mathrm{Nm}^{-1}\right]}\end{array}$ & $\begin{array}{c}c_{a} \\
{\left[\mathrm{Nsm}^{-1}\right]}\end{array}$ & $\begin{array}{c}m_{a} \\
{[\mathrm{~kg}]}\end{array}$ & $\begin{array}{c}\text { maximal displacement } \\
{[\mathrm{mm}]}\end{array}$ \\
\hline- & no TMD & - & - & - & - & 3.447 \\
\hline 1 & simplified & 5 & 86300 & 772 & 600 & 1.349 \\
\hline \multirow[t]{2}{*}{2} & numerical optimization & 5 & 84100 & 1259 & 600 & 1.103 \\
\hline & & 19 & 26000 & 98 & 200 & \\
\hline \multirow[t]{3}{*}{3} & simplified & 5 & 29100 & 150 & 200 & 1.543 \\
\hline & & 27 & 31500 & 129 & 200 & \\
\hline & & 19 & 26800 & 213 & 208 & \\
\hline \multirow[t]{3}{*}{4} & numerical optimization & 5 & 24500 & 198 & 157 & 1.100 \\
\hline & & 27 & 33500 & 312 & 236 & \\
\hline & & 5 & 29800 & 191 & 189 & \\
\hline \multirow[t]{2}{*}{5} & numerical optimization & 5 & 21600 & 147 & 176 & 0.920 \\
\hline & & 5 & 34000 & 391 & 235 & \\
\hline
\end{tabular}

TABLE 3. Comparison of design methods and peak response between 1.7-2.2 Hz.

it was decided to run the optimization multiple times from one hundred randomly generated initial points, which fulfilled the constraints. This procedure allowed us to find good results, but it is not possible to say that the global minimum was found.

\subsection{RESULTS}

The results are summarized in Table 3. A total of 5 different TMD settings were found using simplified method and numerical optimization. As expected, TMDs designed using Den Hartog's criteria were able to reduce the vibration though they were originally proposed for structure with one degree of freedom. It also can be noticed in Figure 6 that single TMD can positively affect vibration of adjacent mode shapes if their frequencies are close enough, and if the adjacent mode shapes have sufficiently high ordinate in the position of TMD.

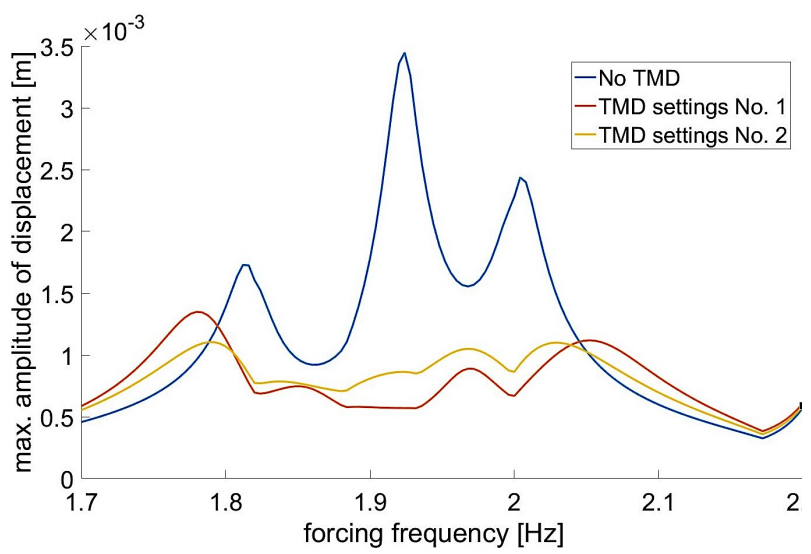

FiguRE 6. FRF - settings No. 1-2.

As is shown in Figures 6 and 7 the numerical optimization was able to improve the performance of both single and multiple TMDs. In the case when starting parameters of TMDs were randomly chosen, we expected that each tuned mass damper would converge to a point where it is able to damp one of the

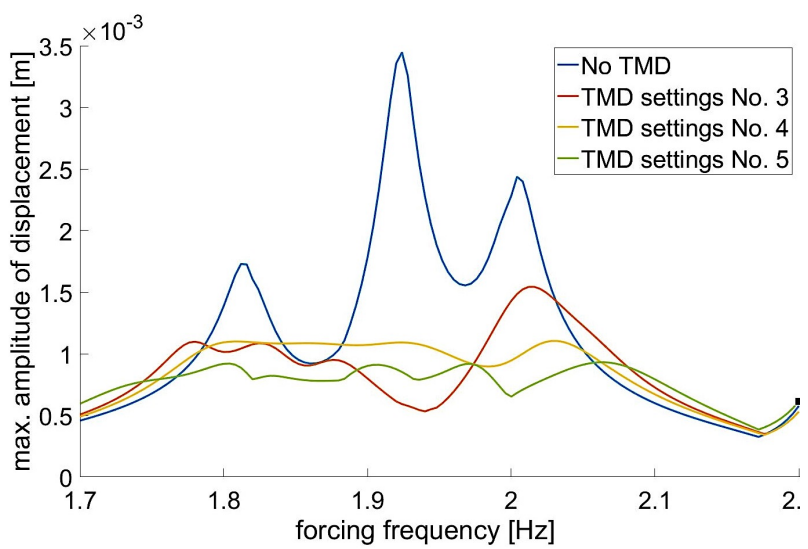

Figure 7. FRF - settings No. 3-5.

dominant frequencies. This assumption was confirmed and can be seen in Figure 8, which shows the FRFs of tuned mass dampers.

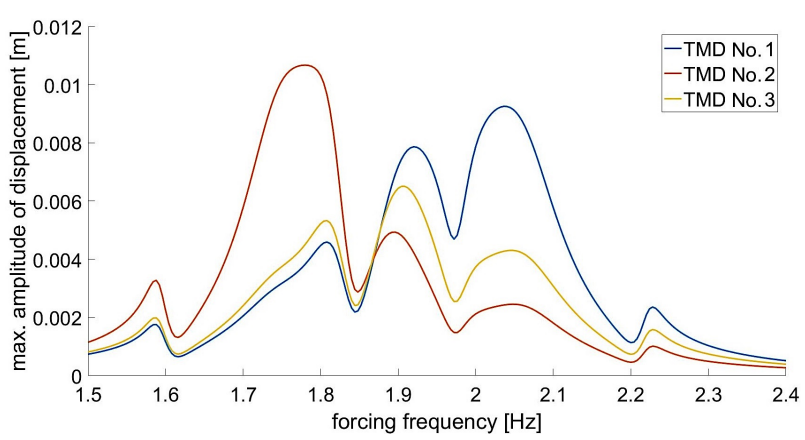

Figure 8. displacement of TMDs - settings No. 5.

Unexpectedly, the best results were provided by settings where all three TMDs were placed in node 5 , despite the fact that this is not the place where the highest ordinates of some mode shapes are located. This phenomenon can be explained by closer observation of mode shapes in Figures 3,4 , and 5 The force is placed in node 5 , therefore all the active mode shapes oscillate with the same phase in this 
node. However, in nodes 19 and 27, the sixth mode shape oscillates in opposite phase than mode shapes 5 and 7 . Therefore neither node 19 nor node 27 are the best place for TMD. However, this situation is closely connected to the fact that the optimization was performed to reduce vibration caused by only one specific force. For design of this footbridge, symmetric placement of smaller TMDs to both sides of the bridge would solve the problem.

\section{CONCLUSIONS}

Modified mode superposition method was used to evaluate response of structure with tuned mass dampers to speed up the response calculation. Utilization of this method provided a powerful tool for numerical optimization, which requires numerous evaluations of steady state response. This response evaluation method provides several important advantages in comparison to standard response calculation:

- Reduction of numerical complexity without noticeable error thanks to reduced number of mode shapes used.

- Allows for a fast evaluation of various load vectors, because the system receptance matrix is known.

- Provides possibility to use experimentally measured mode shapes and natural frequencies for more precise response estimate.

- Can be used for both single and multiple TMDs.

Simplified TMD design method proposed by Den Hartog was compared to numerical optimization. The results demonstrate that numerical design is more appropriate for MDOF structures and can improve the overall performance of TMDs. In all cases of comparison, the analysis showed that optimal damping is higher than the one designed by simplified criterion. Further it was shown that multiple TMDs with the same sum of mass as a single TMD can provide better results.

The best settings of TMDs was able to reduce the peak response between $1.7-2.2 \mathrm{~Hz}$ by $73 \%$, with TMDs total mass of only $0.15 \%$ of the structural mass. This result shows that the common recommendations for TMDs to have 1-3\% of mass of the structure may lead to uneconomical design.

\section{ACKNOWLEDGEMENTS}

The authors gratefully acknowledge support from the Czech Technical University in Prague, project SGS19/032/OHK1/1T/11 Development and application of numerical algorithms for analysis and modeling in mechanics of structures and materials.

\section{REFERENCES}

[1] J. P. Den Hartog. Mechanical Vibrations. McGraw Hill, New York, 1934.

[2] J. Brock. A note on the damped vibration absorber. Journal of Applied Mechanics 68(A):284, 1946.

[3] SETRA. Footbridges: Assessment of vibrational behaviour of footbridges under pedestrian loading, 2006.

[4] E. Caetano, A. Cunha, W. Hoorpah, J. Raoul. Footbridge Vibration Design. CRC Press, 2009.

[5] FIB Bulletin 32: Guidelines for the design of footbridges, 2005.

[6] O. Nishihara, T. Asami. Closed-form exact solution to $\mathrm{h}$ infinity optimization of dynamic vibration absorber: Ii. development of an algebraic approach and its application to a standard problem. Proceedings of the SPIE, 3989, 2000. DOI:10.1117/12.384589.

[7] I. M. Abubakar, B. J. M. Farid. Generalized den hartog tuned mass damper system for control of vibrations in structures. Earthquake Resistant Engineering Structures 104:185-193, 2009.

[8] R. Rana, T. Soong. Parametric study and simplified design of tuned mass dampers. Engineering Structures 20(3):193-204, 1998.

[9] M. B. Ozer, T. J. Royston. Extending den hartog's vibration absorber technique to multi-degree-of-freedom systems. Journal of Vibration and Acoustics 127(4):341-350, 2005. DOI:10.1115/1.1924642

[10] J. Štěpánek, J. Máca. Dynamic response of structures with tuned mass dampers in modal coordinates. Vibroengineering PROCEDIA 23:13-17, 2019. DOI:10.21595/vp.2019.20672 